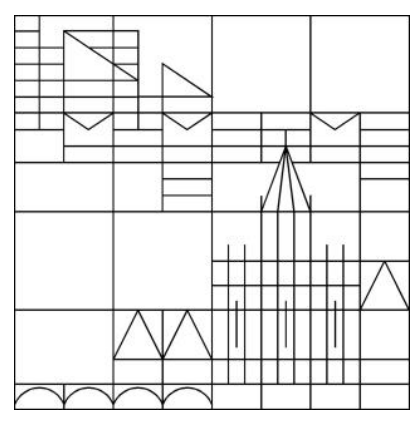

Characterization, perturbation, and interval property of certain sign regular matrices

\author{
Mohammad Adm \\ Jürgen Garloff
}

Konstanzer Schriften in Mathematik

Nr. 395, Dezember 2020

ISSN 1430-3558

Konstanzer Online-Publikations-System (KOPS)

URL: http://nbn-resolving.de/urn:nbn:de:bsz:352-2-1818z0x5djfh87

(C) Fachbereich Mathematik und Statistik

Universität Konstanz

Fach D 197, 78457 Konstanz, Germany 



\title{
Characterization, perturbation, and interval property of certain sign regular matrices
}

\author{
Mohammad Adm ${ }^{\mathrm{a}}$, Jürgen Garloff $\mathrm{b}, \mathrm{c}$ \\ ${ }^{a}$ Department of Applied Mathematics and Physics, Palestine Polytechnic University, \\ Hebron, Palestine \\ ${ }^{b}$ Institute for Applied Research, University of Applied Sciences / HTWG Konstanz, \\ D-78462 Konstanz, Germany \\ ${ }^{c}$ Department of Mathematics and Statistics, University of Konstanz, Konstanz, \\ D-78464 Konstanz, Germany
}

\begin{abstract}
The class of square matrices of order $n$ having a negative determinant and all their minors up to order $n-1$ nonnegative is considered. A characterization of these matrices is presented which provides an easy test based on the Cauchon algorithm for their recognition. Furthermore, the maximum allowable perturbation of the entry in position $(2,2)$ such that the perturbed matrix remains in this class is given. Finally, it is shown that all matrices lying between two matrices of this class with respect to the checkerboard ordering are contained in this class, too.
\end{abstract}

Keywords: Sign regular matrix, totally nonnegative matrix, interval property, checkerboard ordering, Cauchon algorithm

2010 MSC: $15 \mathrm{~A} 48$

\section{Introduction}

A real matrix is called sign regular and strictly sign regular if all its minors of the same order have the same sign or vanish and are nonzero and have the same sign, respectively. Sign regular matrices have found a variety of applications, e.g., in computer aided geometric design [22] and computer vision [20, Section 3.3]. If the sign of all minors of any order is nonnegative (nonpositive) then the matrix is called totally nonnegative (totally nonpositive). Totally nonnegative matrices arise in a variety of ways 
in mathematics and its applications. For background information the reader is referred to the monographs [10], [12], [17], [23].

In [4], we apply the Cauchon algorithm [15], [19] to totally nonnegative matrices and prove a long standing conjecture [13] posed by the second author on intervals of nonsingular totally nonnegative matrices, cf. Theorem 5.1 (ii) below: The underlying ordering is the checkerboard ordering which is obtained from the usual entry-wise ordering in the set of the square real matrices of fixed order by reversing the inequality sign for each entry in a checkerboard fashion. Then all matrices lying between two nonsingular totally nonnegative matrices with respect to this ordering are nonsingular and totally nonnegative, too. The motivation for considering such an interval property stems, e.g., from the investigation of systems of linear equations, where the coefficients of the matrix and the right-hand side are due to uncertainties, e.g., measurement errors, which can be bounded from above and below. Then it is important to know whether all element matrices from the resulting matrix interval have a certain property. For background information the reader is referred to the survey article [14].

In this paper, we continue our study of the Cauchon algorithm and apply it to the class of sign regular matrices having all their minors nonnegative with the exception of the determinant which is negative, termed below $N S T N^{-}$matrices. To the best of our knowledge, such matrices were for the first time more thoroughly investigated in [16], where a characterization and a bidiagonal factorization are presented. The nonsingular $T N$ matrices constitute a subclass of the $P$-matrices which are matrices having all their principal minors positive. The $N s T N^{-}$matrices whose minors of order $n-1$

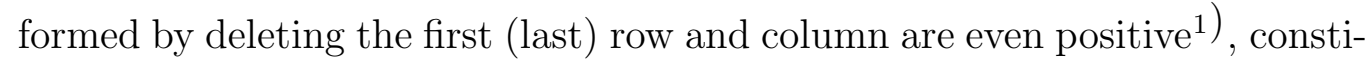
tute a subclass of the almost $P$-matrices, which are matrices having all their proper principal minors positive and a negative determinant. Such matrices originated in the work of Ky Fan [11] and are closely connected with the linear complementarity problem [21]. In [9, Theorems 4.3 and 4.5] and [11, Lemma 3], three subclasses of the almost $P$-matrices are presented which possess the interval property. Another class related to the $N s T N^{-}$matrices are the nonsingular totally nonpositive matrices which are matrices having all their minors nonpositive: If $A$ is $N s T N^{-}$and $S$ the diagonal matrix $\operatorname{diag}(1,-1,1,-1, \ldots)$, then $S A^{-1} S$ is totally nonpositive [16, p.1247]. For

\footnotetext{
1) See Theorem 4.3 (v) below
} 
references and the interval property of these matrices see [6].

In this paper, we employ the Cauchon algorithm to provide further properties of the $N s T N^{-}$matrices and show that these matrices possess the interval property, i.e., all matrices lying between two such matrices with respect to the checkerboard ordering are $N s T N^{-}$matrices, too.

The organization of our paper is as follows. In Section 2, we introduce our notation and give some auxiliary results which we use in the subsequent sections. In Section 3, we recall from [5] the condensed form of the Cauchon algorithm on which our proofs heavily rely. In Section 4, we apply the Cauchon algorithm to derive a new necessary and sufficient condition for a matrix to be $N s T N^{-}$. Also, we present the maximum allowable perturbation of the entry in position $(2,2)$ of an $N s T N^{-}$matrix such that the perturbed matrix remains to be $N s T N^{-}$. In Section 5, we prove the interval property for $N s T N^{-}$matrices and related classes of sign regular matrices.

\section{Notation and auxilary results}

\subsection{Notation}

We now introduce the notation used in our paper. For $\kappa, n$, we denote by $Q_{\kappa, n}$ the set of all strictly increasing sequences of $\kappa$ integers chosen from $\{1,2, \ldots, n\}$. If $\alpha \in Q_{k, n}$, then $\alpha_{\hat{k}}$ denotes the sequence $\alpha$ without its $k$ th member. The dispersion of $\alpha$, denoted by $d(\alpha)$, is defined to be

$$
d(\alpha)=\alpha_{\kappa}-\alpha_{1}-(\kappa-1) ;
$$

it represents a measure for the gaps in the sequence $\alpha$. If $d(\alpha)=0$, i.e., $\alpha$ is formed from consecutive integers, $\alpha$ is called contiguous. We use the set theoretic symbols $\cup, \cap$, and \ to denote somewhat not precisely but intuitively the union, intersection, and difference of two index sequences, where we consider the resulting sequence as strictly increasing ordered. Similarly, we employ the symbol $\epsilon$ to denote membership in a sequence. Let $A$ be a real $n \times n$ matrix. For $\alpha=\left(\alpha_{1}, \alpha_{2}, \ldots, \alpha_{\kappa}\right), \beta=\left(\beta_{1}, \beta_{2}, \ldots, \beta_{\kappa}\right) \in Q_{\kappa, n}$, we denote by $A[\alpha \mid \beta]$ the $\kappa \times \kappa$ submatrix of $A$ contained in the rows indexed by $\alpha_{1}, \alpha_{2}, \ldots, \alpha_{\kappa}$ and columns indexed by $\beta_{1}, \beta_{2}, \ldots, \beta_{\kappa}$. If instead these rows are removed from $A$, we denote the submatrix of $A$ by $A(\alpha \mid \beta]$; we use the notation $A[\alpha \mid \beta)$ if the columns indexed by $\beta$ are deleted. The matrix $A(\alpha \mid \beta)$ is then the matrix obtained from $A$ by deletion of its rows indexed by $\alpha$ and its columns indexed by $\beta$. If $\alpha=\beta$, we denote the principal submatrices of $A$ by $A[\alpha]$ and $A(\alpha)$. We suppress the brackets when we enumerate 
the indices explicitly. If $\alpha=(1, \ldots, n)$, we write $A[-\mid \beta]$ and $A[-\mid \beta)$ and if $\beta=(1, \ldots, n)$, we use the notation $A[\alpha \mid-]$ and $A(\alpha \mid-]$. If $d(\alpha)=d(\beta)=0$, we call the submatrix $A[\alpha \mid \beta]$ as well as its determinant contiguous. For any contiguous $\kappa$-by- $\kappa$ submatrix $A[\alpha \mid \beta]$ of $A$, we call the submatrix

$$
A\left[\alpha_{1}, \ldots, \alpha_{\kappa}, \alpha_{\kappa}+1, \ldots, n \mid 1, \ldots, \beta_{1}-1, \beta_{1}, \ldots, \beta_{\kappa}\right]
$$

of $A$ having $A[\alpha \mid \beta]$ in its upper right corner the left shadow of $A[\alpha \mid \beta]$, and, analogously, we call the submatrix

$$
A\left[1, \ldots, \alpha_{1}-1, \alpha_{1}, \ldots, \alpha_{\kappa} \mid \beta_{1}, \ldots, \beta_{\kappa}, \beta_{\kappa}+1, \ldots, n\right]
$$

having $A[\alpha \mid \beta]$ in its lower left corner the right shadow of $A[\alpha \mid \beta]$.

Let $\epsilon=\left(\epsilon_{1}, \ldots, \epsilon_{n}\right)$ be a signature sequence, i.e., $\epsilon \in\{1,-1\}^{n}$. The matrix $A$ is called strictly sign regular (abbreviated $S S R$ henceforth) and sign regular (abbreviated $S R$ ) with signature $\epsilon$ if $0<\epsilon_{\kappa} \operatorname{det} A[\alpha \mid \beta]$ and $0 \leq \epsilon_{\kappa} \operatorname{det} A[\alpha \mid \beta]$, respectively, for all $\alpha, \beta \in Q_{\kappa, n}, \kappa=1,2, \ldots, n$. If $A$ is $S S R[S R]$ with signature $\epsilon=(1,1, \ldots, 1)$, then $A$ is called totally positive (abbreviated $T P)$ [totally nonnegative (abbreviated $T N)]$. If $A$ is $S R$ with signature $\epsilon=(1, \ldots, 1,-1)$, then we denote this class of matrices by $T N^{-}$. If $A$ is in a certain class of $S R$ matrices and in addition also nonsingular then we affix $N s$ to the name of the class. By $E_{i j}$ we denote the matrix having a 1 in position $(i, j)$ and all other entries zero. We reserve throughout the notation $T_{n}=\left(t_{i j}\right)$ for the backward identity matrix with $t_{i j}:=\delta_{n+1-i, j}, i, j=1, \ldots, n$, and denote $A^{\#}:=T_{n} A T_{n}$. As in [10, p. 34] we obtain that if $A$ is $N s T N^{-}$ then so is $A^{\#}$.

We endow $\mathbb{R}^{n, n}$, the set of the real $n \times n$ matrices, with two partial orderings: Firstly, with the usual entry-wise ordering $\left(A=\left(a_{i j}\right), B=\left(b_{i j}\right) \in\right.$ $\left.\mathbb{R}^{n, n}\right)$

$$
A \leq B: \Leftrightarrow a_{i j} \leq b_{i j}, i, j=1, \ldots, n .
$$

The strict inequality $A<B$ is also understood entry-wise.

Secondly, with the checkerboard ordering, which is defined as follows. Let $S:=\operatorname{diag}\left(1,-1, \ldots,(-1)^{n+1}\right)$ and $A^{*}:=S A S$. Then we define

$$
A \leq^{*} B: \Leftrightarrow A^{*} \leq B^{*} \text {. }
$$

\subsection{Auxiliary results}

Lemma 2.1. [8, Corollary 1] Let $A \in \mathbb{R}^{n, n}$ be such that $\operatorname{det} A[\gamma \mid \delta] \neq 0$, where $\gamma, \delta \in Q_{k, n}$ and let $B=\left(b_{i j}\right)$ be the matrix obtained from $A$ by setting

$$
b_{i j}:=\frac{\operatorname{det} A[\gamma \cup\{i\} \mid \delta \cup\{j\}]}{\operatorname{det} A[\gamma \mid \delta]}, \quad \text { for all } \quad \begin{array}{ll}
i \in\{1, \ldots, n\} \backslash \gamma \text { and } \\
& j \in\{1, \ldots, n\} \backslash \delta .
\end{array}
$$


Then it holds that

$$
\operatorname{rank} A\left[\gamma \cup \gamma^{\prime} \mid \delta \cup \delta^{\prime}\right]=|\gamma|+\operatorname{rank} B\left[\gamma^{\prime} \mid \delta^{\prime}\right]
$$

where $\gamma^{\prime}, \delta^{\prime}$ are strictly increasing sequences from $\{1, \ldots, n-k\}$ and $\gamma \cap \gamma^{\prime}=$ $\delta \cap \delta^{\prime}=\phi$.

Lemma 2.2. [1, Lemma 1.7] Let $A \in \mathbb{R}^{n, m}, \alpha=\left(\alpha_{1}, \ldots, \alpha_{l}\right) \in Q_{l, n}$ and $\beta=\left(\beta_{1}, \ldots, \beta_{l-1}\right) \in Q_{l-1, m-1}$ with $d(\beta)>0$. Then for all $\eta$ such that $\beta_{l-1}<\eta \leq m, k \in\{1, \ldots, l\}, s \in\{1, \ldots, h\}$, and $\beta_{h}<t<\beta_{h+1}$ for some $h \in\{1, \ldots, l-2\}$ or $\beta_{l-1}<t<\eta$ the following determinantal identity holds:

$$
\begin{aligned}
\operatorname{det} A\left[\alpha_{\hat{k}} \mid \beta_{\hat{s}} \cup\{t\}\right] \operatorname{det} A[\alpha \mid \beta \cup\{\eta\}] & =\operatorname{det} A\left[\alpha_{\hat{k}} \mid \beta_{\hat{s}} \cup\{\eta\}\right] \operatorname{det} A[\alpha \mid \beta \cup\{t\}] \\
& +\operatorname{det} A\left[\alpha_{\hat{k}} \mid \beta\right] \operatorname{det} A\left[\alpha \mid \beta_{\hat{s}} \cup\{t, \eta\}\right] .
\end{aligned}
$$

Lemma 2.3. [17, Corollary 9.1], [23, Theorem 1.13] All principal minors of an NsTN matrix are positive.

Lemma 2.4. [23, Proposition 1.15] If $A \in \mathbb{R}^{n, m}$ is $T N$ and rank $A[i+$ $1, \ldots, i+r \mid j+1, \ldots, j+r]=r-1$, then

(i) either the rows $i+1, \ldots, i+r$ or the columns $j+1, \ldots, j+r$ of $A$ are linearly dependent, or

(ii) the right or left shadow of $A[i+1, \ldots, i+r \mid j+1, \ldots, j+r]$ has rank $r-1$.

Theorem 2.5. [16, Theorem 4] Let $A \in \mathbb{R}^{n, n}$. Then $A$ is $N s T N^{-}$if and only if

$$
\left\{\begin{array}{l}
\operatorname{det} A<0, \quad \operatorname{det} A(1) \geq 0, \\
\operatorname{det} A[1, \ldots, k \mid \beta] \geq 0, \quad \beta \in Q_{k, n}, \quad k=1, \ldots, n-1, \\
\operatorname{det} A[\alpha \mid 1, \ldots, k] \geq 0, \quad \alpha \in Q_{k, n}, \quad k=1, \ldots, n-1, \\
\operatorname{det} A[1, \ldots, k]>0, \quad k=1, \ldots, n-2 .
\end{array}\right.
$$

Lemma 2.6. [7, Theorem 2.1] Let $A \in \mathbb{R}^{n, m}$ be of rank $r$ and $\epsilon$ be a signature sequence. If $0 \leq \epsilon_{k} \operatorname{det} A[\alpha \mid \beta]$ for all $\alpha, \beta \in Q_{k, n^{\prime}}$, where $n^{\prime}=\min \{n, m\}$, is valid whenever $d(\beta) \leq n-r$, then $A$ is $S R$ with signature $\epsilon$.

Lemma 2.7. [18, Corollary 3.5] Let $A, B, Z \in \mathbb{R}^{n, n}, A$ and $B$ be nonsingular, and $A \leq Z \leq B$. If $A^{-1}, B^{-1} \geq 0$, then $Z$ is nonsingular and $B^{-1} \leq Z^{-1} \leq$ $A^{-1}$. 


\section{Cauchon diagrams and the Cauchon Algorithm}

In this section we first recall from [15], [19] the definition of a Cauchon diagram and from [5] the condensed form of the Cauchon algorithm.

Definition 3.1. An $n \times m$ Cauchon diagram $C$ is an $n \times m$ grid consisting of $n \cdot m$ squares colored black and white, where each black square has the property that either every square to its left (in the same row) or every square above it (in the same column) is black.

We denote by $C_{n, m}$ the set of the $n \times m$ Cauchon diagrams. We fix positions in a Cauchon diagram in the following way: For $C \in C_{n, m}$ and $i \in\{1, \ldots, n\}, j \in\{1, \ldots, m\},(i, j) \in C$ if the square in row $i$ and column $j$ is black. Here we use the usual matrix notation for the $(i, j)$ position in a Cauchon diagram, i.e., the square in $(1,1)$ position of the Cauchon diagram is in its top left corner.

Definition 3.2. Let $A \in R^{n, m}$ and let $C \in C_{n, m}$. We say that $A$ is a Cauchon matrix associated with the Cauchon diagram $C$ if for all $(i, j)$, $i \in\{1, \ldots, n\}, j \in\{1, \ldots, m\}$, we have $a_{i j}=0$ if and only if $(i, j) \in C$. If $A$ is a Cauchon matrix associated with an unspecified Cauchon diagram, we just say that $A$ is a Cauchon matrix.

Algorithm 3.3. (Condensed form of the Cauchon Algorithm) [5, Algorithm 3.2] Let $A=\left(a_{i j}\right) \in \mathbb{R}^{n, m}$. Set $A^{(n)}:=A$.

For $k=n-1, \ldots, 1$ define $A^{(k)}=\left(a_{i j}^{(k)}\right) \in \mathbb{R}^{n, m}$ as follows:

For $i=1, \ldots, k$,

for $j=1, \ldots, m-1$,

set $s_{j}:=\min \left\{h \in\{j+1, \ldots, m\} \mid a_{k+1, h}^{(k+1)} \neq 0\right\}$ (set $s_{j}:=\infty$ if this set is void),

$$
a_{i j}^{(k)}:= \begin{cases}a_{i j}^{(k+1)}-\frac{a_{k+1, j}^{(k+1)} a_{i s_{j}}^{(k+1)}}{a_{k+1, s_{j}}^{(k+1)}} & \text { if } s_{j}<\infty, \\ a_{i j}^{(k+1)} & \text { if } s_{j}=\infty,\end{cases}
$$

and for $i=k+1, \ldots, n, j=1, \ldots, m$, and $i=1, \ldots, k, j=m, a_{i j}^{(k)}:=a_{i j}^{(k+1)}$. Put $\tilde{A}:=A^{(1)}$.

Lemma 3.4. [15], [4] The matrix $A$ is $T N$ if and only if $\tilde{A}$ is an entry-wise nonnegative Cauchon matrix. $A$ is in addition nonsingular if and only if all diagonal entries of $\tilde{A}$ are positive. 
We recall from [19] the definition of a lacunary sequence associated with a Cauchon diagram.

Definition 3.5. Let $C \in \mathcal{C}_{n, m}$. We say that a sequence

$$
\gamma:=\left(\left(i_{k}, j_{k}\right), \quad k=0,1, \ldots, t\right)
$$

which is strictly increasing in both arguments is a lacunary sequence with respect to $C$ if the following conditions hold:

(i) $\left(i_{k}, j_{k}\right) \notin C, k=1, \ldots, t$;

(ii) $(i, j) \in C$ for $i_{t}<i \leq n$ and $j_{t}<j \leq m$.

(iii) Let $s \in\{1, \ldots, t-1\}$. Then $(i, j) \in C$ if

(a) either for all $(i, j), i_{s}<i<i_{s+1}$ and $j_{s}<j$, or for all $(i, j), i_{s}<i<i_{s+1}$ and $j_{0} \leq j<j_{s+1}$

and

(b) either for all $(i, j), i_{s}<i$ and $j_{s}<j<j_{s+1}$ or for all $(i, j), i<i_{s+1}$, and $j_{s}<j<j_{s+1}$.

Proposition 3.6. [2, Corollary 3.3] Let $A \in \mathbb{R}^{n, m}$ be such that $\tilde{A}$ is a Cauchon matrix and let $\gamma=\left(\left(i_{k}, j_{k}\right), k=0,1, \ldots, t\right)$ be a lacunary sequence. Then the following representation holds

$$
\operatorname{det} A\left[i_{0}, i_{1}, \ldots, i_{t} \mid j_{0}, j_{1}, \ldots, j_{t}\right]=\tilde{a}_{i_{0}, j_{0}} \cdot \tilde{a}_{i_{1}, j_{1}} \cdot \ldots \cdot \tilde{a}_{i_{t}, j_{t}} .
$$

\section{A necessary and sufficient condition for a matrix to be $N s T N^{-}$}

Lemma 4.1. Let $A \in \mathbb{R}^{n, n}$ be such that $A(n), A[-\mid 1)$, and $A(1 \mid-]$ are $T N$, and $A(n \mid 1)$ and $A(1, n)$ are nonsingular. Then the following minors are nonnegative

$$
\begin{aligned}
& \operatorname{det} A[1, \ldots, \ell \mid \beta \cup\{n\}], \\
& \operatorname{det} A[\beta \cup\{n\} \mid 1, \ldots, \ell],
\end{aligned}
$$

where $\ell=1, \ldots, n-1$, and $\beta=\left(\beta_{1}, \ldots, \beta_{\ell-1}\right) \in Q_{\ell-1, n-1}$ with $\beta_{1}=1$.

Proof. We only prove (5a). The proof of (5b) is similar. For $\ell=1$, $\operatorname{det} A[1, \ldots, \ell \mid \beta \cup$ $\{n\}]=a_{1 n}$ which is nonnegative since $A[-\mid 1)$ is $T N$. Assume $2 \leq \ell \leq n-1$ 
and let $\alpha=(1, \ldots, \ell), \beta_{1}=1$. Then by Lemma 2.2 , we obtain choosing $k=s=1$ and $\eta=n$

$\left.\operatorname{det} A\left[2, \ldots, \ell \mid \beta_{\hat{1}} \cup\{t\}\right)\right] \operatorname{det} A[1, \ldots, \ell \mid \beta \cup\{n\}]=\operatorname{det} A\left[2, \ldots, \ell \mid \beta_{\hat{1}} \cup\{n\}\right] \times$ $\operatorname{det} A[1, \ldots, \ell \mid \beta \cup\{t\}]+\operatorname{det} A[2, \ldots, \ell \mid \beta] \operatorname{det} A\left[1, \ldots, \ell \mid \beta_{\hat{1}} \cup\{t, n\}\right]$,

for all $t \in\{1, \ldots, n-1\} \backslash \beta$. The minors on the right-hand side and $\left.\operatorname{det} A\left[2, \ldots, \ell \mid \beta_{\hat{1}} \cup\{t\}\right)\right]$ are nonnegative since they correspond to minors in $A(1 \mid-], A[-\mid 1)$, and $A(n)$, for any $t \in\{1, \ldots, n-1\} \backslash \beta$. If for all $\ell=$ $2, \ldots, n-1$, there exists $t_{\ell} \in\{1, \ldots, n-1\} \backslash \beta$ such that $\operatorname{det} A\left[2, \ldots, \ell \mid \beta_{\hat{1}} \cup\right.$ $\left.\left.\left\{t_{\ell}\right\}\right)\right]>0$, then we are done. Otherwise, for some $\ell \in\{2, \ldots, n-1\}$, $\left.\operatorname{det} A\left[2, \ldots, \ell \mid \beta_{\hat{1}} \cup\{t\}\right)\right]=0$ for all $t \in\{1, \ldots, n-1\} \backslash \beta$. Hence $A[2, \ldots, \ell \mid 2, \ldots, n-$ 1] has rank at most $\ell-2$. By Lemma 2.3, we have for $\ell=2, \ldots, n-2$, $\operatorname{det} A[2, \ldots, \ell \mid 3, \ldots, \ell]$ are positive since $A(n \mid 1)$ is $N s T N$ and by the assumption $\operatorname{det} A(1, n)>0$. Hence $A[2, \ldots, \ell \mid 2, \ldots, n-1]$ has rank $\ell-1$ for all $\ell=2, \ldots, n-1$ which is a contradiction. Hence for each $\ell=2, \ldots, n-1$, there exists $t_{\ell} \in\{1, \ldots, n-1\} \backslash \beta$ such that $\left.\operatorname{det} A\left[2, \ldots, \ell \mid \beta_{\hat{1}} \cup\left\{t_{\ell}\right\}\right)\right]>0$. Hence for $\ell=1, \ldots, n-1$, $\operatorname{det} A[1, \ldots, \ell \mid \beta \cup\{n\}] \geq 0$.

Lemma 4.2. Let $A \in \mathbb{R}^{n, n}$ be $N s T N^{-}$. Then the following minors are positive:

(i) For any $\alpha \in Q_{k, n}$ such that $k \leq n-2$, $\operatorname{det} A[\alpha]>0$;

(ii) for $r=1, \ldots, n-1$,

$\operatorname{det} A[2, \ldots, r+1 \mid 1, \ldots, r]>0, \operatorname{det} A[1, \ldots, r \mid 2, \ldots, r+1]>0$,

$\operatorname{det} A[r+1, \ldots, n \mid r, \ldots, n-1]>0, \operatorname{det} A[r, \ldots, n-1 \mid r+1, \ldots, n]>0 .(7)$

In particular,

$$
\operatorname{det} A(1 \mid n), \quad \operatorname{det} A(n \mid 1)>0
$$

Proof. Let $A$ be $N s T N^{-}$. Assume (i) is not true. Then there exists $\alpha \in Q_{k, n}$ such that $k \leq n-2$ and $\operatorname{det} A[\alpha]=0$. Assume that $k_{0}$ is the smallest $k$, $1 \leq k \leq n-2$, such that statement (i) is not true. If $k_{0}=1$, then assume that $a_{i i}=0$. Since $A$ is nonsingular there must be nonzero entries in row $i$ and column $i$. By considering minors of order 2 it follows that the left or the right shadow of $a_{i i}$ is the nullmatrix which implies that $A$ is singular. In 
the following assume $1<k_{0}$. Then there exists $\alpha=\left(\alpha_{1}, \ldots, \alpha_{k_{0}}\right) \in Q_{k_{0}, n}$ such that $\operatorname{det} A[\alpha]=0$ and $\operatorname{det} A\left[\alpha_{\hat{i}_{0}}\right]>0$ for some $i_{0} \in\left\{1, \ldots, k_{0}\right\}$. Set $\gamma=\delta:=\alpha_{\hat{i}_{0}}$ and define $B$ as in Lemma 2.1. Since $A$ is $N s T N^{-}$we have $B$ is so. Moreover, $b_{i_{0}, i_{0}}=0$ which implies that $B$ is singular. Hence by Lemma 2.1, $A$ is also singular which is a contradiction. This completes proof of (i). To show (ii), we first prove (8). We apply Sylvester's Determinant Identity, e.g., [10, pp 29 - 30] to obtain

$$
\operatorname{det} A(1, n) \operatorname{det} A=\operatorname{det} A(1) \operatorname{det} A(n)-\operatorname{det} A(1 \mid n) \operatorname{det} A(n \mid 1) .
$$

By (i), $\operatorname{det} A(1, n)$ is positive. If $\operatorname{det} A(1 \mid n) \operatorname{det} A(n \mid 1)=0$, then it follows that

$$
\operatorname{det} A=\frac{\operatorname{det} A(1) \operatorname{det} A(n)}{\operatorname{det} A(1, n)} \geq 0
$$

which is a contradiction. Hence $\operatorname{det} A(1 \mid n)>0$ and $\operatorname{det} A(n \mid 1)>0$.

The remaining inequalities are now a simple consequence of (8) and Lemma 2.3 .

Inequalities (6) are shown in [16, Lemma 2] by a lengthy and complicated proof.

Theorem 4.3. Let $A \in \mathbb{R}^{n, n}$ be $N s T N^{-}$and let $\tilde{A}$ be the matrix obtained from $A$ by the application of Algorithm 3.3. Then the following statements hold:

(i) $\tilde{A}[-\mid 1)$ and $\tilde{A}(1 \mid-]$ are nonnegative Cauchon matrices;

(ii) $\tilde{a}_{i, i+1}, \tilde{a}_{i+1, i}>0, i=1, \ldots, n-1$;

(iii) $\tilde{a}_{i i}>0, i=3, \ldots, n$.

(iv) If $\tilde{a}_{22}>0$, then $\tilde{a}_{11}<0$.

(v) $\tilde{a}_{22}=0$ if and only if $\operatorname{det} A(1)=0$.

Proof. (i) By the fact that the matrices that are obtained by the application of Algorithm 3.3 to the submatrices $A[-\mid 1)$ and $A(1 \mid-]$ coincide with the matrices $\tilde{A}[-\mid 1)$ and $\tilde{A}(1 \mid-]$, respectively, and $A$ is $N s T N^{-}$, we have by Lemma 3.4 that the matrices are nonnegative Cauchon matrices. 
(ii) By (i), $\tilde{A}[-\mid 1)$ and $\tilde{A}(1 \mid-]$ are nonnegative Cauchon matrices. By (8) and proceeding by decreasing induction with respect to the lexicographic order starting from position $(n-1, n)$ and $(n, n-1)$, respectively, the following sequences are lacunary with respect to $C_{\tilde{A}[-\mid 1)}$ and $C_{\tilde{A}(1 \mid-]}$

$$
\begin{aligned}
& ((i, i+1),(i+1, i+2), \ldots,(n-1, n)), \\
& ((i+1, i),(i+2, i+1), \ldots,(n, n-1)),
\end{aligned}
$$

$i=1, \ldots, n-1$. Therefore, by Proposition 3.6 and (8) we have $\tilde{a}_{i, i+1}>0$ and $\tilde{a}_{i+1, i}>0$.

(iii) We proceed as in (ii) noticing that $((i, i),(i+1, i+1), \ldots,(n, n))$, $i=3, \ldots, n$, are lacunary sequences with respect to $C_{\tilde{A}(1 \mid-]}$ and using Lemma 4.2 (i).

(iv) By (i) and since $\tilde{a}_{22}>0, \tilde{A}$ is a nonnegative Cauchon matrix with except possibly $\tilde{a}_{11}$. Since the sequence $((1,1),(2,2), \ldots,(n, n))$ is a lacunary sequence with respect to $C_{\tilde{A}}$, we obtain by Proposition 3.6 that $\operatorname{det} A=\tilde{a}_{11} \cdot \tilde{a}_{22} \cdot \ldots \cdot \tilde{a}_{n n}$. Therefore, by (iii) and $\tilde{a}_{22}>0$ we conclude that $\tilde{a}_{11}<0$.

(v) By (i) and (iii), the sequence $((2,2),(3,3), \ldots,(n, n))$ is a lacunary sequence with respect to $C_{\tilde{A}(1 \mid-]}$. Hence by Proposition 3.6, $\operatorname{det} A(1)$ can be represented as

$$
\operatorname{det} A(1)=\tilde{a}_{22} \cdot \tilde{a}_{33} \cdot \ldots \cdot \tilde{a}_{n n}
$$

and we can conclude by (iii) that

$$
\tilde{a}_{22}=\frac{\operatorname{det} A(1)}{\tilde{a}_{33} \cdot \ldots \cdot \tilde{a}_{n n}} .
$$

Therefore, $\operatorname{det} A(1)=0$ if and only if $\tilde{a}_{22}=0$.

The preceding results allow us now to present a sufficient criterion based on Algorithm 3.3 for a real matrix to be $N s T N^{-}$.

Theorem 4.4. Let $A \in \mathbb{R}^{n, n}$ be such that (i)-(iv) hold in Theorem 4.3, and let $\operatorname{det} A(n)$ be nonnegative. Then $A$ is $N s T N^{-}$. 
Proof. We obtain by Theorem 4.3 (i) and arguing similarly as in its proof that $A[-\mid 1)$ and $A(1 \mid-]$ are $T N$. Since $\tilde{a}_{i, i+1}, \tilde{a}_{i+1, i}>0, i=1, \ldots, n-1$, the sequences $((1,2),(2,3), \ldots,(n-1, n))$ and $((2,1),(3,2), \ldots,(n, n-1))$ are lacunary with respect to $C_{\tilde{A}[-\mid 1)}$ and $C_{\tilde{A}(1 \mid-]}$, respectively. Hence by Proposition 3.6 we have $\operatorname{det} A(n \mid 1)>0$ and $\operatorname{det} A(1 \mid n)>0$. Moreover, by Lemma 2.3 we have $\operatorname{det} A(1, n \mid 1,2)>0$ and $\operatorname{det} A(1,2 \mid 1, n)>0$. In the same way we may conclude from (iii) that $\operatorname{det} A[3, \ldots, n]>0$ and by Lemma 2.3 it follows that $\operatorname{det} A[3, \ldots, n-1]>0$. Therefore, if $\operatorname{det} A(1, n)=0$, then rank $A(1, n)=n-3$. By considering $A(1, n)$ as a submatrix with rank $n-3$ in the $T N$ matrix $A(1 \mid-]$ we have by Lemma 2.4 that the rows $2,3, \ldots, n-1$ or the columns $2,3, \ldots, n-1$ are linearly dependent or the left or the right shadow of $A(1, n)$ has rank $n-3$ which contradicts that $\operatorname{det} A(1, n \mid 1,2)>0$ and $\operatorname{det} A(1,2 \mid 1, n)>0$. Therefore, we obtain

$$
\operatorname{det} A(1, n)>0
$$

Let $B:=A(n)$.

Claim. $B$ is $T N$.

The submatrices $B[-\mid 1)$ and $B(1 \mid-]$ are TN and and the matrices that are obtained by the application of Algorithm 3.3 to these submatrices coincide with the matrices $\tilde{B}[-\mid 1)$ and $\tilde{B}(1 \mid-]$, respectively. Application of Algorithm 3.3 to $B$ yields $\tilde{B}$ with all its entries nonnegative with the exception of possibly $\tilde{b}_{11}$. Moreover, we obtain by Lemma $3.4, \tilde{b}_{k k}>0, k=2, \ldots, n-1$, since $B(1)=A(1, n)$ is $N s T N$ and the application of Algorithm 3.3 to the submatrix $B(1)$ coincides with the submatrix $\tilde{B}(1)$. Furthermore, $\tilde{B}$ is a Cauchon matrix: if $\tilde{b}_{i j}=0$ for some $i, j=2, \ldots, n-1$, then all of the entries to its left in the same row must be zero whenever $i>j$ or the entries above it in the same column must be zero whenever $i<j$ since $B(1 \mid-]$ and $B[-\mid 1)$ are $T N$ and $\tilde{b}_{k k}>0, k=2, \ldots, n-1$. The sequence $((1,1),(2,2), \ldots,(n-$ $1, n-1))$ is a lacunary sequence with respect to $C_{\tilde{B}}$. Hence by Proposition 3.6 , det $B$ can be represented as $\operatorname{det} B=\tilde{b}_{11} \cdot \tilde{b}_{22} \cdot \ldots \cdot \tilde{b}_{n-1, n-1}$. Therefore, we obtain

$$
\tilde{b}_{11}=\frac{\operatorname{det} B}{\tilde{b}_{22} \cdot \ldots \cdot \tilde{b}_{n-1, n-1}}=\frac{\operatorname{det} A(n)}{\tilde{b}_{22} \cdot \ldots \cdot \tilde{b}_{n-1, n-1}} \geq 0 .
$$

Whence $\tilde{B}$ is a nonnegative Cauchon matrix and by Lemma $3.4, B$ is $T N$. To show $\operatorname{det} A<0$, we distinguish the following two cases:

Case 1. $\tilde{a}_{22}>0$.

In this case, $\tilde{A}$ is a Cauchon matrix and the sequence $((1,1),(2,2), \ldots,(n, n))$ 
is a lacunary with respect to $C_{\tilde{A}}$. Hence by Proposition $3.6, \operatorname{det} A$ can be represented as $\operatorname{det} A=\tilde{a}_{11} \cdot \tilde{a}_{22} \cdot \ldots \cdot \tilde{a}_{n n}$, and we conclude by the assumption (iv) that $\operatorname{det} A<0$.

Case 2. $\tilde{a}_{22}=0$.

We proceed as in proof of (v) in Theorem 4.3 to conclude that $\operatorname{det} A(1)=0$ since $\tilde{a}_{22}=0$. By Sylvester's Determinant Identity, we obtain

$$
\begin{aligned}
\operatorname{det} A(1, n) \operatorname{det} A & =\operatorname{det} A(1) \operatorname{det} A(n)-\operatorname{det} A(1 \mid n) \operatorname{det} A(n \mid 1) \\
& =-\operatorname{det} A(1 \mid n) \operatorname{det} A(n \mid 1) .
\end{aligned}
$$

Because $\operatorname{det} A(1 \mid n), \operatorname{det} A(n \mid 1), \operatorname{det} A(1, n)>0$, we conclude from the last equality that $\operatorname{det} A<0$.

To conclude the proof, by Lemma 2.6 it is enough to show that for all $\alpha, \beta \in$ $Q_{\ell, n}, \ell=1, \ldots, n-1, \operatorname{det} A[\alpha \mid \beta] \geq 0$ with $d(\alpha)=0$. If $\alpha_{1}>1$ or $\beta_{1}>1$ or $\alpha_{1}=1$ and $\beta_{\ell}<n$, then $\operatorname{det} A[\alpha \mid \beta] \geq 0$ since $A[-\mid 1), A(1 \mid-]$, and $A(n)$ are $T N$. Hence we are left to show that the following minors are nonnegative

$$
\operatorname{det} A[1, \ldots, \ell \mid \beta]
$$

where $\ell=1, \ldots, n-1$, and $\beta=\left(\beta_{1}, \ldots, \beta_{\ell}\right) \in Q_{\ell, n-1}$ with $\beta_{1}=1$ and $\beta_{\ell}=n$. By Lemma 4.1 such minors are nonnegative. Therefore, $A$ is $N s T N^{-}$.

Theorems 4.3 and 4.4 together provide a necessary and sufficient condition for a matrix $A \in \mathbb{R}^{n, n}$ to be $N s T N^{-}$. This provides a test which requires only to run Algorithm 3.3 twice (one time in order to check the sign of $\operatorname{det} A(n)$ ). It is an alternative to the test based on [16, Theorem 8] which relies on Neville elimination and bidiagonal factorization. Both tests require $O\left(n^{3}\right)$ operations.

The analysis made so far shows that the coefficient in position $(2,2)$ in an $N s T N^{-}$matrix is the most critical one. In the following two theorems we consider the invariance of the property of being $N s T N^{-}$of the matrix $A$ under perturbations of $a_{22}$.

Theorem 4.5. Let $A \in \mathbb{R}^{n, n}$ be $N s T N^{-}$and let $\delta^{-}:=\min \left\{\frac{\operatorname{det} A(n)}{\operatorname{det} A(2, n)}, \frac{\operatorname{det} A(1)}{\operatorname{det} A(1,2)}\right\}$. Then for any $x \in\left[0, \delta^{-}\right]$,

$$
B_{x}:=A-x E_{22}
$$

is $N s T N^{-}$. The bound $\delta^{-}$is the best possible. 
Proof. Let $x$ be any real number in $\left[0, \min \delta^{-}\right]$. By Theorem 4.4, it is enough to show that $\tilde{B}_{x}=\left(\tilde{b}_{i j}\right)$ satisifies (i) - (iv) in Theorem 4.3 and $\operatorname{det} B_{x}(n) \geq 0$, where $\tilde{B}_{x}$ is the matrix obtained by the application of Algorithm 3.3 to $B_{x}$. By the fact that the matrices that are obtained by the application of Algorithm 3.3 to the submatrices $B_{x}[-\mid 1)$ and $B_{x}(1 \mid-]$ coincide with the matrices $\tilde{B}_{x}[-\mid 1)$ and $\tilde{B}_{x}(1 \mid-]$, respectively, and

$$
\left.\begin{array}{rl}
\tilde{b}_{i+1, i+1} & =\tilde{a}_{i+1, i+1}>0, \\
\tilde{b}_{i, i+1} & =\tilde{a}_{i, i+1}>0, \\
\tilde{b}_{i+1, i} & =\tilde{a}_{i+1, i}>0,
\end{array}\right\} \text { for } i=2, \ldots, n-1,
$$

we have that $\tilde{B}_{x}[-\mid 1)$ and $\tilde{B}_{x}(1 \mid-]$ are nonnegative Cauchon matrices with except possibly $\tilde{b}_{12}, \tilde{b}_{21}$, and $\tilde{b}_{22}$. By $(15)$, the sequences $((1,2),(2,3), \ldots,(n-$ $1, n)),((2,1),(3,2), \ldots,(n, n-1))$, and $((2,2),(3,3), \ldots,(n, n))$ are lacunary with respect to $C_{\tilde{B}_{x}(1 \mid-]}$ and $C_{\tilde{B}_{x}[-\mid 1)}$. Hence by Proposition 3.6, we obtain

$$
\tilde{b}_{12}=\frac{\operatorname{det} B_{x}(n \mid 1)}{\tilde{b}_{23} \cdot \ldots \cdot \tilde{b}_{n-1, n}}=\frac{\operatorname{det} A(n \mid 1)+x \operatorname{det} A(2, n \mid 1,2)}{\tilde{a}_{23} \cdot \ldots \cdot \tilde{a}_{n-1, n}}>0,
$$

and similarly $\tilde{b}_{21}>0$. The inequality

$$
\tilde{b}_{22}=\frac{\operatorname{det} B_{x}(1)}{\tilde{b}_{33} \cdot \ldots \cdot \tilde{b}_{n n}}=\frac{\operatorname{det} A(1)-x \operatorname{det} A(1,2)}{\tilde{a}_{33} \cdot \ldots \cdot \tilde{a}_{n, n}} \geq 0,
$$

is valid if and only if $x \leq \frac{\operatorname{det} A(1)}{\operatorname{det} A(1,2)}$. Moreover, if $\tilde{b}_{22}>0$, then $\tilde{B}_{x}$ is a Cauchon matrix and the sequence $((1,1), \ldots,(n, n))$ is lacunary with respect to $C_{\tilde{B}_{x}}$ and by Proposition 3.6, we obtain

$$
\tilde{b}_{11}=\frac{\operatorname{det} B_{x}}{\tilde{b}_{22} \cdot \ldots \cdot \tilde{b}_{n n}}=\frac{\operatorname{det} A-x \operatorname{det} A(2)}{\tilde{b}_{22} \cdot \ldots \cdot \tilde{b}_{n, n}}<0 .
$$

Finally,

$$
\operatorname{det} B_{x}(n)=\operatorname{det} A(n)-x \operatorname{det} A(2, n)=\operatorname{det} A(2, n)\left(\frac{\operatorname{det} A(n)}{\operatorname{det} A(2, n)}-x\right) .
$$

Hence $\operatorname{det} B_{x}(n) \geq 0$ if and only if $x \leq \frac{\operatorname{det} A(n)}{\operatorname{det} A(2, n)}$. Therefore, $B_{x}$ is $N s T N^{-}$ for all $x \in\left[0, \min \delta^{-}\right]$. 
Theorem 4.6. Let $A \in \mathbb{R}^{n, n}$ be $N s T N^{-}$and let $\mathcal{S}^{+}$be the set of defined quantities among $-\frac{\operatorname{det} A}{\operatorname{det} A(2)}, \frac{\operatorname{det} A(n \mid 1)}{\operatorname{det} A(2, n \mid 1,2)}, \frac{\operatorname{det} A(1 \mid n)}{\operatorname{det} A(1,2 \mid 2, n)}$. Define $\delta^{+}$as follows:

$$
\delta^{+}:= \begin{cases}\min \mathcal{S}^{+} & \text {if } \mathcal{S}^{+} \text {is not void } \\ \infty & \text { otherwise. }\end{cases}
$$

Then for any $x \in\left[0, \delta^{+}\right)$,

$$
B_{x}:=A+x E_{2,2}
$$

is $N$ sT $N^{-}$. The bound $\delta^{+}$is the best possible.

Proof. By Lemma $4.2, \delta^{+}$is positive. In the following we may suppose that $x>0$. Firstly, we show that $D:=B_{x}[-\mid 1)$ is $T N$ for all $0<x<\frac{\operatorname{det} A(n \mid 1)}{\operatorname{det} A(2, n \mid 1,2)}$ if $\operatorname{det} A(2, n \mid 1,2) \neq 0$ and for all $x>0$ if $\operatorname{det} A(2, n \mid 1,2)=0$. We proceed by showing that $\tilde{D}$ is a nonnegative Cauchon matrix (which is obtained from $D$ by Algorithm 3.3). By the definition of $D$ and Lemma 4.2 we have for $k=2, \ldots, n-1$

$$
\operatorname{det} D[k, \ldots, n-1]=\operatorname{det} A[k, \ldots, n-1 \mid k+1, \ldots, n]>0,(17)
$$

$\operatorname{det} D[k+1, \ldots, n \mid k, \ldots, n-1]=\operatorname{det} A[k+1, \ldots, n]>0$.

Therefore, the matrices $D[k, \ldots, n-1]$ and $D[k+1, \ldots, n \mid k, \ldots, n-1]$ are $N s T N$ and by Lemma 3.4, the matrices obtained from both matrices by Algorithm 3.3 are Cauchon matrices with positive diagonal entries from which we can form lacunary sequences. By Proposition 3.6, we get for $k=2, \ldots, n-$ 1 the following representation of entries of $\tilde{D}$

$$
\begin{aligned}
\tilde{d}_{k k} & =\frac{\operatorname{det} D[k, k+1, \ldots, n-1]}{\operatorname{det} D[k+1, \ldots, n-1]} \\
\tilde{d}_{k+1, k} & =\frac{\operatorname{det} D[k+1, k+2, \ldots, n \mid k, k+1, \ldots, n-1]}{\operatorname{det} D[k+2, \ldots, n \mid k+1, \ldots, n-1]} .
\end{aligned}
$$

By (19), (20), $A[-\mid 1)$ is $T N$, and by the fact that $\tilde{D}(1,2 \mid-]$ and $\tilde{D}[-\mid 1)$ coincide with the matrices that are obtained by the application of Algorithm 3.3 to the submatrices $D(1,2 \mid-]$ and $D[-\mid 1)$, respectively, we conclude that $\tilde{D}$ is a Cauchon matrix with all of its entries are nonnegative with exception of possibly $\tilde{d}_{11}$ and $\tilde{d}_{21}$. Since $((1,1),(2,2), \ldots,(n-1, n-1))$ and $((2,1),(3,2), \ldots,(n, n-1))$ are lacunary sequences with respect to $C_{\tilde{D}}$, we 
obtain the following representation of $\tilde{d}_{11}$ and $\tilde{d}_{21}$ from which we conclude that

$$
\begin{aligned}
\tilde{d}_{11} & =\frac{\operatorname{det} D[1, \ldots, n-1]}{\operatorname{det} D[2, \ldots, n-1]}=\frac{\operatorname{det} A(n \mid 1)-x \operatorname{det} A(2, n \mid 1,2)}{\operatorname{det} A(1, n \mid 1,2)}, \\
\tilde{d}_{21} & =\frac{\operatorname{det} D[2, \ldots, n \mid 1, \ldots, n-1]}{\operatorname{det} D[3, \ldots, n \mid 2, \ldots, n-1]}=\frac{\operatorname{det} A(1)+x \operatorname{det} A(1,2)}{\operatorname{det} A(1,2)}
\end{aligned}
$$

Therefore, $\tilde{D}$ is a nonnegative matrix if and only if $\operatorname{det} A(n \mid 1)-x \operatorname{det} A(2, n \mid 1,2)>$ 0 which is equivalent to $0<x<\frac{\operatorname{det} A(n \mid 1)}{\operatorname{det} A(2, n \mid 1,2)}$ if $\operatorname{det} A(2, n \mid 1,2) \neq 0$ and $x>0$ if $\operatorname{det} A(2, n \mid 1,2)=0$. In the same way we can show that $B_{x}(1 \mid-]$ is $T N$ for all $0<x<\frac{\operatorname{det} A(1 \mid n)}{\operatorname{det} A(1,2 \mid 2, n)}$ if $\operatorname{det} A(1,2 \mid 2, n) \neq 0$ and $x>0$ if $\operatorname{det} A(1,2 \mid 2, n)=0$.

We conclude the proof by showing that for any $x \in\left(0, \delta^{+}\right), B_{x}$ is $N s T N^{-}$. If $\operatorname{det} A(2)=0$ or $0<x<-\frac{\operatorname{det} A}{\operatorname{det} A(2)}$ if $\operatorname{det} A(2) \neq 0$, we obtain by Laplace expansion along the second column that $\operatorname{det} B_{x}<0$. Let $\alpha, \beta \in Q_{k, n}, k=$ $1, \ldots, n-1$. In the following, we show that $\operatorname{det} B_{x}[\alpha \mid \beta] \geq 0$. We distinguish the following two cases:

Case 1. $2 \notin \alpha \cap \beta$.

In this case we have $\left.\operatorname{det} B_{x}[\alpha \mid \beta]=\operatorname{det} A_{[} \alpha \mid \beta\right] \geq 0$.

Case 2. $2 \in \alpha \cap \beta$.

If in addition $1 \in \alpha \cap \beta$, then we have

$$
\operatorname{det} B_{x}[\alpha \mid \beta]=\operatorname{det} A[\alpha \mid \beta]+x \operatorname{det} A\left[\alpha_{\hat{2}} \mid \beta_{\hat{2}}\right] \geq 0 \text {. }
$$

Otherwise, $1 \notin \alpha$ or $1 \notin \beta$ which implies that $B_{x}[\alpha \mid \beta]$ is a submatrix in $B_{x}(1 \mid-]$ or $B_{x}[-\mid 1)$, respectively. Therefore, $\operatorname{det} B_{x}[\alpha \mid \beta]$ is nonnegative since $B_{x}(1 \mid-]$ and $B_{x}[-\mid 1)$ are $T N$.

\section{Example 4.7. Let}

$$
A=\left[\begin{array}{ccc}
1 & 1 & 1 \\
1 & 1.75 & 3 \\
1 & 3 & 6
\end{array}\right]
$$

which is $N s T N^{-}$and $B_{x}:=A+x E_{22}, x \in \mathbb{R}$. Then $\operatorname{det} B_{x}=5 x-0.25$ and $\operatorname{det} B_{x}(1)=1.5+6 x$. If $x=0.05=\frac{-\operatorname{det} A}{\operatorname{det} A(2)}$, $\operatorname{det} B_{x}$ changes its sign from - to + , whereas the other minors remain positive, and at $-x=0.25=$ $\frac{\operatorname{det} A(1)}{\operatorname{det} A(1,2)}$, det $B_{x}(1)$ changes its sign from + to - , whereas the other minors keep their sign. So the interval $[-0.25,0.05)$ provides the maximum allowable perturbation such that $B_{x}$ remains $N s T N^{-}$. 


\section{The interval property}

In this section, we show that the class of $N s T N^{-}$matrices possesses the interval property. We will make use of the interval property of the $N s T N$ matrices. For a weakening of the nonsingularity assumption in (ii), see [3].

Theorem 5.1. [4, Lemma 3.2, Theorem 3.6, and Corollary 3.7] Let A, B, Z $\in$ $\mathbb{R}^{n, n}$ be such that $A \leq^{*} Z \leq^{*} B$ and let $A$ and $B$ be $T N$.

(i) If $A(1)$ or $A(n)$ is nonsingular, then $Z$ is $T N$.

(ii) If $A$ is nonsingular, then $Z$ is $N s T N$.

Now we are in the position to state the interval property of $N s T N^{-}$ matrices.

Theorem 5.2. Let $A, B, Z \in \mathbb{R}^{n, n}$ be such that $A \leq^{*} Z \leq^{*} B$ and let $A$ and $B$ be $N s T N^{-}$. Then $Z$ is $N s T N^{-}$, too.

Proof. We show that the intermediate matrix $Z$ fulfils the conditions of Theorem 2.5. Since $A \leq * B$, we have $S(-B) S \leq S(-A) S$, where $S=$ $\operatorname{diag}\left(1,-1,1, \ldots,(-1)^{n-1}\right)$. By $-S A^{-1} S=(S(-A) S)^{-1},-S B^{-1} S=(S(-B) S)^{-1} \geq$ 0 , we may apply Lemma 2.7 , to conclude that

$$
-S Z^{-1} S \geq 0
$$

Application of Lemma 4.2 (i), (8), and Theorem 5.1 (ii) yields that $Z[1, \ldots, n-$ 2], $Z(1 \mid n)$, and $Z(n \mid 1)$ are $N s T N$. By Lemma 4.2 (i) and Theorem 5.1 (i) applied to $A(n)$ and $B(n)$, we conclude that $Z(n)$ is $T N$. Similarly we obtain that $Z(1)$ is $T N$. To prove that $\operatorname{det} Z[1, \ldots, k \mid \beta] \geq 0, \beta \in Q_{k, n}$, $k=1, \ldots, n-1$, we use that the remaining minors are all of the form (5a). To apply Lemma 4.1, we have to show that $Z[-\mid 1)$ and $Z(1 \mid-]$ are $T N$ (for the assumption that $\operatorname{det} A(1, n)>0$ see $(13))$. We only prove that $Z(1 \mid-]$ is $T N$, the proof that $Z[-\mid 1)$ is $T N$ is analogous. Since $Z(1 \mid n)$ is nonsingular, $Z(1 \mid-]$ has rank $n-1$. By the fact that $Z(1 \mid n)$ and $Z(1)$ are $T N$, and by Lemma 2.6, we are left to show that the determinants of the submatrices which are lying in the first and last columns of $Z(1 \mid-]$ and which are formed from consecutive columns with the exception of a gap of one column are all nonnegative. These are the minors $\operatorname{det} Z(1 \mid 1, \ldots, j-1, j+1, \ldots, n]$. The entry in position $(j, 1)$ of $-S Z^{-1} S$ is $\frac{-\operatorname{det} Z(1, j)}{\operatorname{det} Z}$ which is nonnegative by $(23), j=2, \ldots, n-1$. Therefore, $\operatorname{det} Z(1, j) \geq 0$. To show that $\operatorname{det} Z[\alpha \mid 1, \ldots, k] \geq 0, \alpha \in Q_{k, n}$, $k=1, \ldots, n-1$, we proceed similarly. 
Using Theorem 5.2, we obtain the interval property of further classes of $N s S R$ matrices.

Theorem 5.3. Let $A, B, Z \in \mathbb{R}^{n, n}$ be such that $A \leq^{*} Z \leq^{*} B$ and let $A$ and $B$ be NsSR matrices with the same signature $\epsilon=\left(\epsilon_{1}, \ldots, \epsilon_{n}\right)$. If $\epsilon$ is one of the following signatures:

(i) $\epsilon_{i}=(-1)^{i}, i=1, \ldots, n-1, \epsilon_{n}=(-1)^{n-1}$,

(ii) $\epsilon_{i}=(-1)^{\frac{i(i-1)}{2}}, i=1, \ldots, n-1, \epsilon_{n}=(-1)^{\frac{n(n-1)}{2}+1}$,

(iii) $\epsilon_{i}=(-1)^{\frac{i(i+1)}{2}}, i=1, \ldots, n-1, \epsilon_{n}=(-1)^{\frac{n(n+1)}{2}+1}$,

then $Z$ is $N s S R$ with signature $\epsilon$.

Proof. $D:=\operatorname{diag}(-1,-1, \ldots,-1)$ and $T_{n}$ are $N s S R$ matrices with signatures $\epsilon_{i}=(-1)^{i}$ and $\epsilon_{i}=(-1)^{\frac{i(i-1)}{2}}, i=1, \ldots, n$, respectively, and $D^{-1}=D$ and $T_{n}^{-1}=T_{n}$. Hence if $A$ and $B$ are $N s S R$ matrices with the same signature which is given in one of (i)-(iii), then by [7, Theorem 3.1] the following hold. If $\epsilon$ is the signature in case

(i), then $D A$ and $D B$,

(ii), then $T_{n} A$ and $T_{n} B$,

(iii), then $D T_{n} A$ and $D T_{n} B$

are $N s T N^{-}$, and by Theorem 5.2, Z is an $N s S R$ matrix with the same signature.

Conclusion In this paper, we have provided by using the Cauchon algorithm a new characterization of the matrices having all their proper minors nonnegative and a negative determinant, the class $N s T N^{-}$. We have presented the maximum allowable perturbation of the most critical entry of such a matrix such that the perturbed matrix remains in the class. Finally, we have shown that the $N s T N^{-}$matrices possess the interval property. This result provides a further class of nonsingular sign regular matrices which has the interval property [6]. 


\section{References}

[1] M. Adm, Perturbation and Intervals of Totally Nonnegative Matrices and Related Properties of Sign Regular Matrices, Dissertation, University of Konstanz, Konstanz, Germany, 2016.

[2] M. Adm, K. Al Muhtaseb, A. Abedel Ghani, S.M. Fallat, J. Garloff, Further applications of the Cauchon algorithm to rank determination and bidiagonal factorization, Linear Algebra Appl. 545 (2018) 240-255.

[3] M. Adm, K. Al Muhtaseb, A. Abedel Ghani, J. Garloff, Relaxing the nonsingularity assumption for intervals of totally nonnegative matrices, Electron. J. Linear Algebra 36 (2020) 106-123.

[4] M. Adm, J. Garloff, Intervals of totally nonnegative matrices, Linear Algebra Appl. 439 (2013) 3796-3806.

[5] M. Adm, J. Garloff, Improved tests and characterizations of totally nonnegative matrices, Electronic J. Linear Algebra 27 (2014) 588-610.

[6] M. Adm, J. Garloff, Intervals of special sign regular matrices, Linear and Multilinear Algebra 64 (2016) 1424-1444.

[7] T. Ando, Totally positive matrices, Linear Algebra Appl. 90 (1987) 165219.

[8] C. de Boor, A. Pinkus, The approximation of a totally positive band matrix by a strictly banded totally positive one, Linear Algebra Appl. 42 (1982) 81-98.

[9] P.N. Choudhury, M.R. Kannan, Interval hulls of $N$-matrices and almost $P$-matrices, submitted to Linear Algebra Appl., available at arXiv:2009.04405 (Sept. 4, 2020).

[10] S.M. Fallat, C.R. Johnson, Totally Nonnegative Matrices, Princeton Ser. Appl. Math., Princeton University Press, Princeton and Oxford, 2011.

[11] K. Fan, Some matrix inequalities, Abh. Math. Sem. Univ. Hamburg 29 (1966) 185-196. 
[12] F.R. Gantmacher, M.G. Krein, Oscillation Matrices and Kernels and Small Vibrations of Mechanical System, AMS Chelsea Publishing, Providence, RI. Translation based on the 1941 Russian original, 2002.

[13] J. Garloff, Criteria for sign regularity of sets of matrices, Linear Algebra Appl. 44 (1982) 153-160.

[14] J. Garloff, M. Adm, J. Titi, A survey of classes of matrices possessing the interval property and related properties, Reliable Computing 22 (2016) $1-10$.

[15] K.R. Goodearl, S. Launois, T.H. Lenagan, Totally nonnegative cells and matrix Poisson varieties, Adv. Math. 226 (2011) 779-826.

[16] R. Huang, A test and bidiagonal factorization for certain sign regular matrices, Linear Algebra Appl. 438 (2013) 1240-1251.

[17] S. Karlin, Total Positivity, Vol. I., Stanford University Press, Stanford, Calif, 1968.

[18] J.R. Kuttler, A fourth-order finite-difference approximation for the fixed membrane eigenproblem, Math. Comp. 25 (1971) 237-256.

[19] S. Launois, T.H. Lenagan, Efficient recognition of totally nonnegative matrix cells, Found. Comput. Math. 14 (2014) 371-387.

[20] T. Lindeberg, Scale-Space Theory in Computer Vision, Kluwer Acad. Publ., Dordrecht, 1994.

[21] J.M. Miao, Ky Fan's N-matrices and linear complementarity problems, Math. Program. 61 (1993) 351-356.

[22] J.M. Peña, Shape Preserving Representations in Computer-aided Geometric Design. Nova Science Publishers, Commack, NewYork, 1999.

[23] A. Pinkus, Totally Positive Matrices. Cambridge Tracts in Math. 181, Cambridge Univ. Press, Cambridge, UK, 2010. 